\title{
Differential Inhibition of Prion Propagation by Enantiomers of Quinacrine
}

\author{
Chongsuk Ryou, Giuseppe Legname, David Peretz, John C. Craig, \\ Michael A. Baldwin, and Stanley B. Prusiner \\ Institute for Neurodegenerative Diseases (CR, GL, DP, MAB, SBP), Departments of Neurology (GL, DP, MAB, SBP), \\ Pharmaceutical Chemistry (JCC, MAB), and Biochemistry and Biophysics (SBP), University of California, \\ San Francisco, California
}

\begin{abstract}
SUMMARY: Prion diseases are fatal neurologic disorders caused by accumulation of a pathogenic isoform (PrPsc) of the prion protein (PrP). The recent discovery of the inhibitory action of quinacrine on $\mathrm{PrP}^{\mathrm{Sc}}$ formation in scrapie-infected neuroblastoma (ScN2a) cells raised the possibility of a treatment for patients with prion disease. To investigate the efficacy of quinacrine enantiomers, we measured the inhibitory effect of these isomers on $\mathrm{PrP}^{\mathrm{Sc}}$ formation in ScN2a cells. (S)-quinacrine exhibited superior antiprion activity compared with $(R)$-quinacrine and two generic quinacrines that appear to be racemates. Treatment with these various forms of quinacrine did not induce adverse changes affecting cell survival and the expression of marker proteins over a range of potentially therapeutic concentrations. Thus, quinacrine enantiomers demonstrated stereoselectivity on prion elimination but not cytotoxicity in ScN2a cells. Our results raise the possibility that in vivo treatment using one enantiomer of quinacrine may be superior to a racemic mixture, which is the form that is generally used when quinacrine is employed to treat parasitic diseases. (Lab Invest 2003, 83:837-843).
\end{abstract}

\begin{abstract}
Drion diseases, including Creutzfeldt-Jakob dis1 ease (CJD) in humans, scrapie in sheep, and bovine spongiform encephalopathy in cattle, are invariably fatal. Although many compounds have been reported to inhibit prion formation or enhance clearance in cultured cells as well as prolong incubation times in rodents, no compound has been found to reverse prion disease once clinical signs of CNS dysfunction have appeared (Caspi et al, 1998; Caughey and Race, 1992; Dickinson et al, 1975; Diringer and Ehlers, 1991; Ehlers and Diringer, 1984; Kimberlin and Walker, 1983; Peretz et al, 2001; Priola et al, 2000; Supattapone et al, 1999, 2001). Recently, one of us (SBP) as well as another independent group of investigators reported that both acridine and phenothiazine derivatives inhibit formation of the diseaseassociated pathogenic isoform ( $\mathrm{PrPS}$ ) of the prion protein $(\mathrm{PrP})$ in cultured scrapie-infected neuroblastoma (ScN2a) cells (Doh-ura et al, 2000; Korth et al, 2001). In both studies, quinacrine inhibited PrPSc accumulation in ScN2a cells with an effective concentration for half-maximal inhibition $\left(\mathrm{EC}_{50}\right)$ of $0.3 \mu \mathrm{M}$. Exposure to quinacrine for 6 days eliminated $\mathrm{PrP}^{\mathrm{Sc}}$ in ScN2a cells permanently (Korth et al, 2001). Because
\end{abstract}

\section{DOI: 10.1097/01.LAB.0000074919.08232.A2}

Received April 11, 2003.

This work was supported by grants from the National Institutes of Health and by a gift from the G. Harold and Leila Y. Mathers Charitable Foundation.

Address reprint requests to: Dr. Stanley B. Prusiner, Institute for Neurodegenerative Diseases, University of California, Box 0518, San Francisco, California94143-0518.E-mail: stanley@itsa.ucsf.edu quinacrine can penetrate the blood-brain barrier and a wealth of knowledge on the toxicity of quinacrine in humans has been established, these findings therefore prompted some of us to begin evaluating the efficacy of quinacrine in patients dying of CJD (B. Miller, M. Geschwind, S. J. DeArmond, and S. B. Prusiner, unpublished data). To date, our experience and that of others on the efficacy of quinacrine in the treatment of patients with prion disease have yielded mixed results.

Traditionally, the pharmacologic potency and selectivity of optical isomers against many diseases can differ enormously despite their identical physicochemical properties (Albert, 1960; Brocks and Jamali, 1995; Hutt and Tan, 1996). There is considerable interest in switching from a racemate to a single enantiomeric isomer for many currently available drugs (Tucker, 2000).

Quinacrine, first synthesized in the early 1930s, was extensively used as an antimalarial for nearly 2 decades. Because one of us (JCC) had previously studied the antimalarial activity of the two enantiomers, $(S)$ - and (R)-quinacrine (Webster et al, 1991), we explored the stereoselectivity of quinacrine on prion propagation. Because chronic administration of quinacrine is associated with side effects such as liver dysfunction, cardiomyopathy, and toxic psychosis (Findlay, 1951; Goodman and Gilman, 1955), the use of one stereoisomer of quinacrine, if it was proven to be more efficacious than the racemic mixture, might be associated with diminished toxicity.

Here we report that $(S)$-quinacrine eliminates $\operatorname{PrPSc}$ from ScN2a cells more effectively than either $(R)$ - 
quinacrine or a racemic mixture, as demonstrated by Western blot analysis and ELISA results. Over a range of potentially therapeutic concentrations, $(S)$ - and $(R)$ quinacrine neither produced cytotoxicity nor interfered with the expression of other proteins in cells. Although these in vitro results raise the possibility of (S)quinacrine providing a more effective treatment against prion diseases, in vivo toxicologic, pharmacodynamic, and pharmacokinetic data from animal experiments are needed prior to any treatment in humans with a quinacrine enantiomer.

\section{Results}

To measure the antiprion effect of quinacrine enantiomers, we incubated ScN2a cells with generic quinacrine, $(S)$-quinacrine, or $(R)$-quinacrine (Fig. 1) as described previously (Korth et al, 2001). On Western blot analysis, the 6-day incubation of ScN2a cells with 0.1 to $0.4 \mu \mathrm{M}(S)$-quinacrine demonstrated a dosedependent reduction in the accumulation of proteaseresistant $\mathrm{PrP}^{\mathrm{Sc}}$ (Fig. 2A, lanes 2-5). In contrast, a similar reduction in $\mathrm{PrP}^{\mathrm{Sc}}$ levels required $0.6 \mu \mathrm{M}$ of $(R)$-quinacrine (Fig. 2A, lane 6). Incubation at concentrations $>0.6 \mu \mathrm{M}$ of $(S)$-quinacrine completely eliminated proteinase $\mathrm{K}(\mathrm{PK})$-resistant $\mathrm{PrP}^{\mathrm{Sc}}$ (Fig. 2A, lanes 6-8), whereas more than $0.8 \mu \mathrm{M}$ of either $(R)$ quinacrine or generic quinacrine was needed to remove all $\mathrm{PrP}^{\mathrm{Sc}}$ (Fig. 2A, lanes 7-8). The pattern of $\mathrm{PrP}^{\mathrm{Sc}}$ disappearance in cell lysates incubated with $(R)$-quinacrine was similar to that of generic quinacrine used in earlier studies (Sigma Chemical, St. Louis, Missouri) (Fig. 2A). Densitometric analysis shows the $\mathrm{PrP}^{\mathrm{Sc}}$ elimination profiles of $(S)$-quinacrine, $(R)$ quinacrine, and generic quinacrine at various concentrations (Fig. 2B).

In addition to densitometry, the antiprion activity of quinacrine enantiomers was quantified by ELISA measurements of the levels of $\mathrm{PrP}^{\mathrm{Sc}}$ in ScN2a cells (Fig. 3). Our ELISA results are similar to those obtained by Western blotting and its densitometric analysis (Fig. 2). The efficiency of $\mathrm{PrP}^{\mathrm{Sc}}$ reduction by each enantiomer was almost identical at concentrations less than 0.2 $\mu \mathrm{M}$ and greater than $0.8 \mu \mathrm{M}$. However, at concentrations between 0.2 and $0.8 \mu \mathrm{M},(S)$-quinacrine was more efficient in reducing $\mathrm{PrP}^{\mathrm{Sc}}$ levels; at concentrations between 0.3 and $0.6 \mu \mathrm{M}$, the antiprion activity of $(S)$-quinacrine was 2- to 6-fold greater than that of $(R)$-quinacrine.<smiles>CCCCN(CCCN(C)CC)C(C)C</smiles>

$(R)$-quinacrine<smiles>CCCCCCCNc1c2ccc(Cl)cc2nc2ccc(OC)cc12</smiles>

(S)-quinacrine
Figure 1.

Structures of $(R)$-quinacrine and $(S)$-quinacrine.

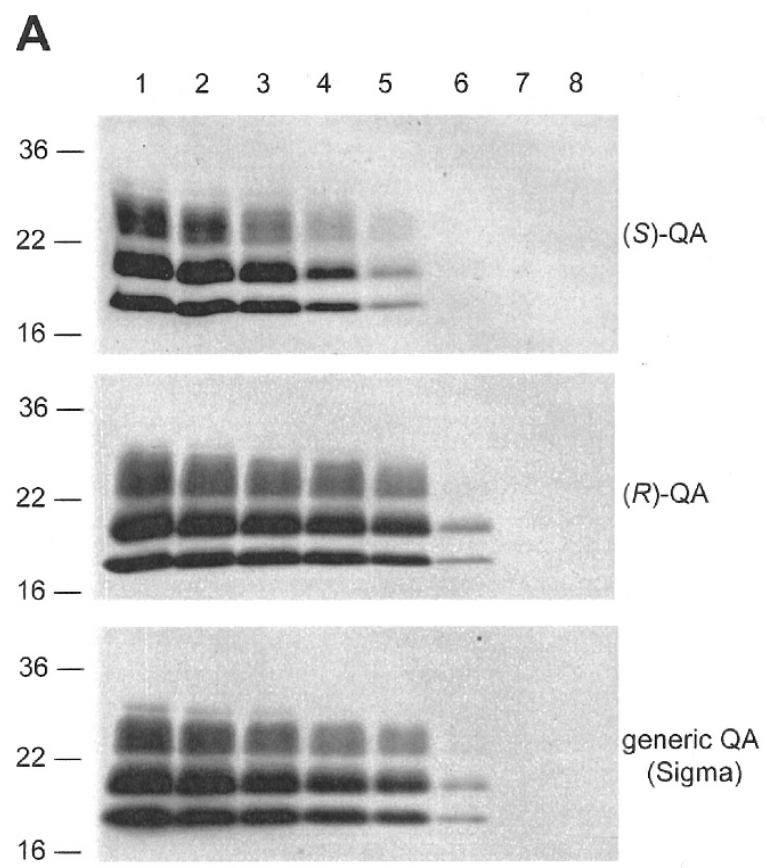

B

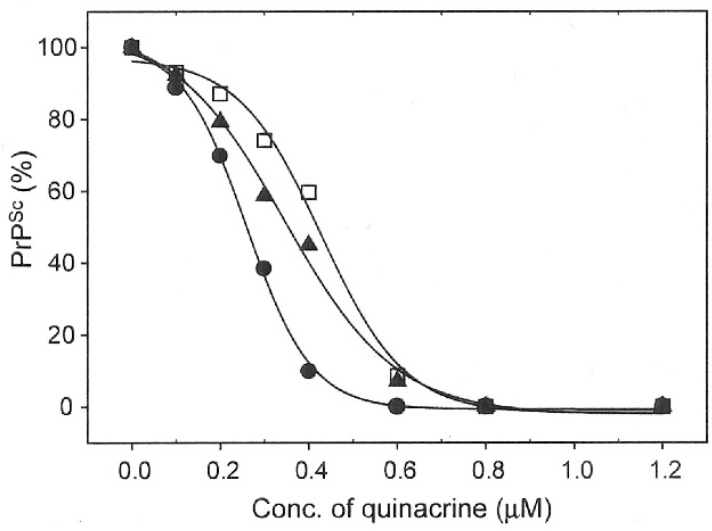

Figure 2.

Differential accumulation of PrPSc in ScN2a cells treated with enantiomers of quinacrine $(Q A)$. A, Western blots of proteinase $\mathrm{K}$-digested ScN2a cell lysates incubated with $(S)$-quinacrine, $(R)$-quinacrine, and a generic mixture of quinacrine from Sigma Chemical. Molecular weight markers $(\mathrm{kDa})$ are shown to the left of each blot. Lanes 1 to 8 represent quinacrine concentrations of 0 $\mu \mathrm{M}, 0.1 \mu \mathrm{M}, 0.2 \mu \mathrm{M}, 0.3 \mu \mathrm{M}, 0.4 \mu \mathrm{M}, 0.6 \mu \mathrm{M}, 0.8 \mu \mathrm{M}$, and $1.2 \mu \mathrm{M}$, respectively. B, Densitometric analysis of panel A. Kodak 1D (Kodak) for densitometry and Sigma Plot (SPSS Science) for mathematical analysis were used. $X$-axis, concentrations of quinacrine $(\mu \mathrm{M}) ; Y$-axis, PrPSc levels (\%). Filled circles, $(S)$-quinacrine; filled triangles, generic quinacrine from Sigma Chemical; open squares, $(R)$-quinacrine.

We also compared the generic quinacrine (Panorama Compounding, Van Nuys, California) currently used for treating patients with prion disease (B. Miller, M. Geschwind, S.J. DeArmond, and S.B. Prusiner, unpublished data) to (S)-quinacrine. As expected, the generic quinacrine was less potent than (S)quinacrine. At a concentration of $0.4 \mu \mathrm{M},(S)$ quinacrine removed almost all $\mathrm{PrP}^{\mathrm{Sc}}$, whereas a concentration of $0.5 \mu \mathrm{m}$ of the generic quinacrine was required for the same effect (Fig. 4A). Densitometric 


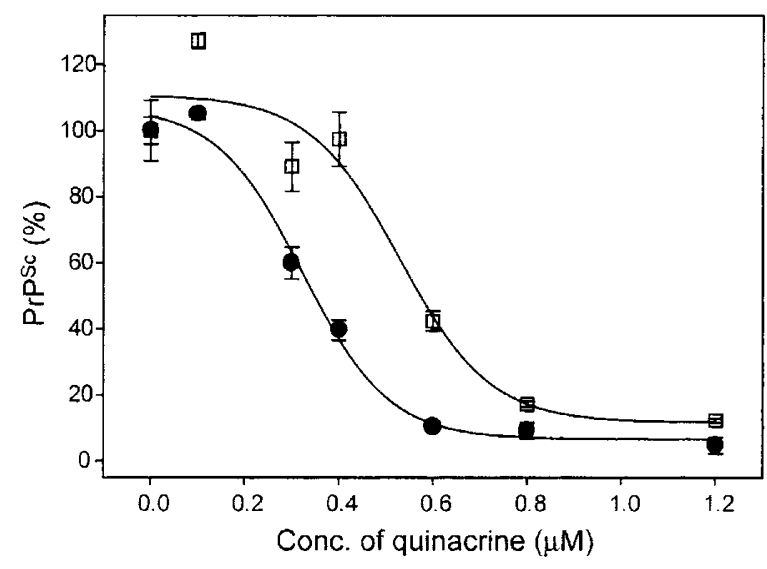

Figure 3.

PrPSc detection by ELISA. ELISA of proteinase K-digested ScN2a cell lysates incubated with $(S)$-quinacrine and $(R)$-quinacrine. Data points and bars represent the mean and standard deviation from three independent experiments. $X$-axis, concentrations of quinacrine $(\mu \mathrm{M}) ; Y$-axis, PrPSc levels (\%). Filled circles, $(S)$-quinacrine; open squares, $(R)$-quinacrine.

analysis showed that the $\mathrm{PrP}^{\mathrm{Sc}}$ inhibition curve for the quinacrine used for treatment of patients with prion disease (Fig. 4B, open triangles) was similar but not identical to that of the quinacrine from Sigma Chemical (Fig. 2B, filled triangles). In lower concentrations $(0.1$ to $0.3 \mu \mathrm{M})$, the quinacrine used for treatment of patients was less effective at eliminating $\mathrm{PrP}^{\mathrm{Sc}}$ than the material from Sigma Chemical.

The effective concentrations for half-maximal inhibition $\left(\mathrm{EC}_{50}\right)$ of $(R)$-quinacrine, $(S)$-quinacrine, and generic quinacrine were calculated from densitometric analysis of Western blots and ELISA determinations. The $\mathrm{EC}_{50}$ values for generic quinacrine obtained from Sigma and Panorama were 0.36 and $0.35 \mu \mathrm{M}$, respectively, intermediate between those of $(S)$ - and $(R)$ quinacrine. The $\mathrm{EC}_{50}$ values of $(S)$ - and $(R)$-quinacrine were 0.25 and $0.44 \mu \mathrm{M}$, respectively, by Western blotting followed by densitometry (Fig. 2B); and 0.31 and $0.58 \mu \mathrm{M}$, respectively, by ELISA (Fig. 3). Our findings suggest that the generic quinacrine from Panorama is a mixture of quinacrine enantiomers that is similar to the generic quinacrine from Sigma.

To test whether various quinacrines used in this study caused alterations that affect cell survival, we conducted two independent assays in the ScN2a cells. Because both total protein and mitochondrial enzyme activity represent cell viability, we measured these changes in the ScN2a cells treated with various quinacrine concentrations for the studies described above. Total protein content in cells treated with quinacrine (racemic and enantiomeric) remained constant (Fig. 5A). Total protein in individual culture dishes totaled 1.0 to $1.2 \mathrm{mg}$. In the MTT (3-[4,5-dimethylthiazol-2-yl]-2,5-diphenyltetrazolium bromide; Sigma) assay, cell viability is indicated by mitochondrial dehydrogenase activity, which is linearly proportional to the measured absorbance of formazan deposited in the living cells. Cells treated with either quinacrine enantiomer exhibited maximum viability at concentrations $<1.5 \mu \mathrm{M}$ (Fig. 5B). The cytotoxicity of both enantiomers was almost equal at most concen-

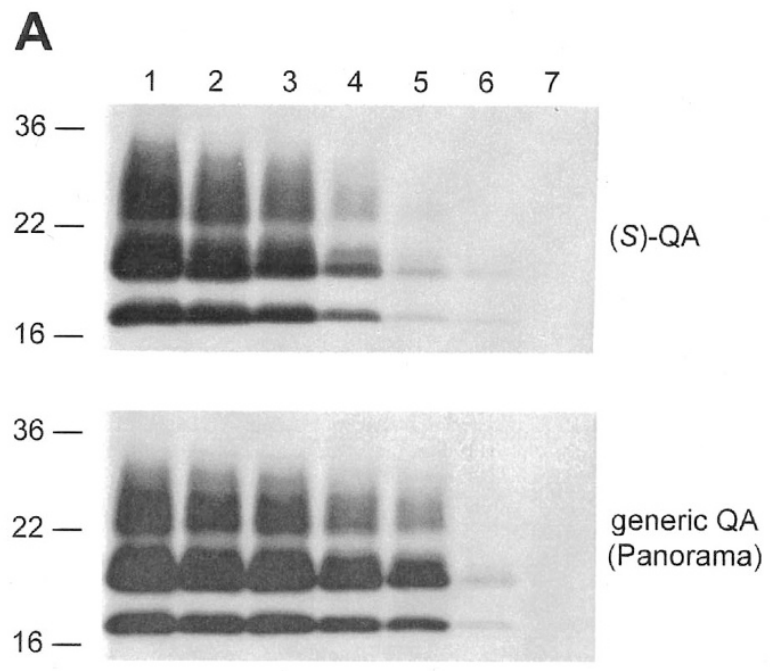

B

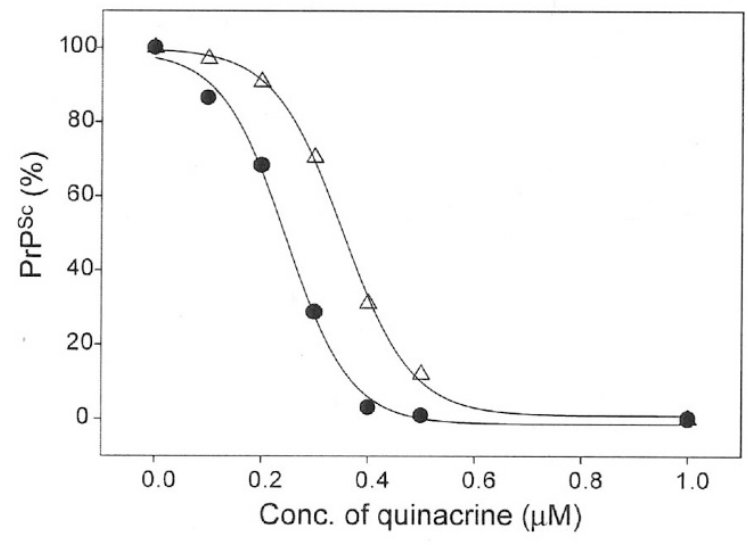

Figure 4.

Comparison of antiprion activity between $(S)$ - and generic quinacrine $(Q A)$. A, Western blots of proteinase K-digested ScN2a cell lysates incubated with (S)-quinacrine and a generic quinacrine from Panorama Compounding. Molecular weight markers $(\mathrm{kDa})$ are shown to the left of each blot. Lanes 1 to 7 represent quinacrine concentrations of $0 \mu \mathrm{M}, 0.1 \mu \mathrm{m}, 0.2 \mu \mathrm{m}, 0.3 \mu \mathrm{M}, 0.4 \mu \mathrm{m}$, $0.5 \mu \mathrm{M}$, and $1.0 \mu \mathrm{M}$, respectively. B, Densitometric analysis of panel A. Kodak 1D (Kodak) for densitometry and Sigma Plot (SPSS Science) for mathematical analysis were used. $X$-axis, concentrations of quinacrine $(\mu \mathrm{M}) ; Y$-axis, PrPSc levels $(\%)$. Filled circles, (S)-quinacrine; open triangles, generic quinacrine from Panorama Compounding.

trations, but $(R)$-quinacrine is less toxic between 1.8 and $2.0 \mu \mathrm{M}$. Although such concentration ranges are beyond our interest, it is noteworthy that $(R)$-quinacrine demonstrated less cytotoxicity than (S)-quinacrine at $2.0 \mu \mathrm{m}$; at concentrations $>2.5 \mu \mathrm{M}$, both enantiomers were equally cytotoxic. The calculated $\mathrm{LC}_{50}$ values (concentration causing half-maximal lethality) of $(S)$ - and $(R)$-quinacrine were 1.98 and $2.24 \mu \mathrm{M}$, respectively (Fig. 5B), which were outside the range of concentrations needed to inhibit $\mathrm{PrP}^{\mathrm{Sc}}$ formation.

In addition to the effects of quinacrine treatment on the survival of ScN2a cells, we also examined changes in tubulin and $\mathrm{PrP}^{\mathrm{C}}$ levels in the cells for alterations that may have been caused by quinacrine treatment. In cells treated with different concentrations of $(S)$ quinacrine, $(R)$-quinacrine, and generic quinacrine, rel- 
A

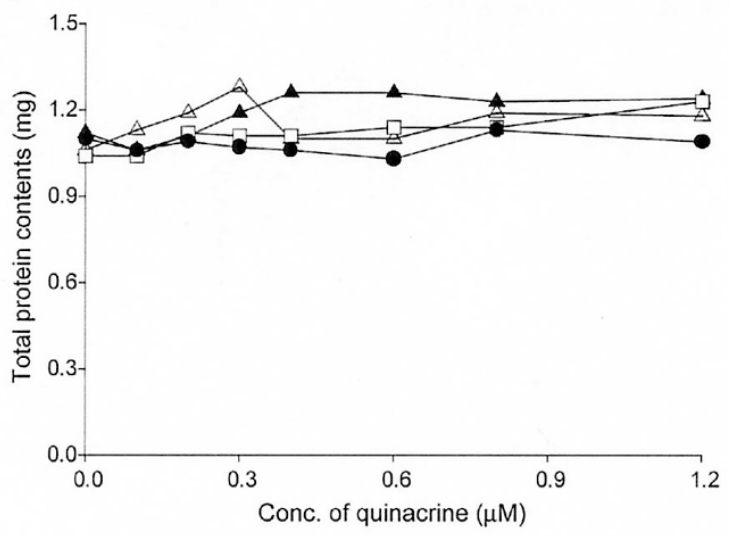

B

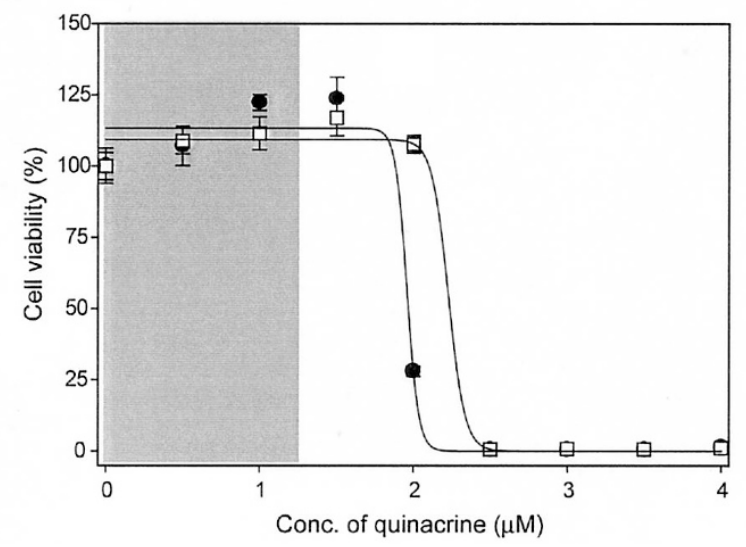

Figure 5.

Effect of quinacrine treatment on total protein and viability in ScN2a cells. A, Changes in total protein contents by quinacrine treatment. Tested concentrations of quinacrine were between 0 and $1.2 \mu \mathrm{m}$. X-axis, concentrations of quinacrine $(\mu \mathrm{M}) ; Y$-axis, total protein contents $(\mathrm{mg})$. Filled circles, $(S)$ quinacrine; filled triangles, generic quinacrine from Sigma Chemical; open triangles, generic quinacrine from Panorama Compounding; open squares, $(R)$-quinacrine. B, Changes in cell viability by quinacrine enantiomers. Tested concentrations of quinacrine were between 0 and $4.0 \mu \mathrm{m}$. The shaded area represents quinacrine concentrations of therapeutic interest. Absorbance of untreated cells was used to normalize values for cell viability. Data points and bars represent the mean and standard deviation from three independent experiments. $X$-axis, concentrations of quinacrine $(\mu \mathrm{M}) ; Y$-axis, cell viability $(\%)$. Filled circles, (S)-quinacrine; open squares, $(R)$-quinacrine. ative ratios indicated that tubulin and $\operatorname{PrP}^{\mathrm{C}}$ levels remained unchanged (Table 1).

\section{Discussion}

In the studies described here, we investigated the antiprion activity and cytotoxicity of quinacrine enantiomers in ScN2a cells. We found that the $(S)$-quinacrine enantiomer showed greater potency in eliminating $\mathrm{PrP}^{\mathrm{Sc}}$ compared with that of $(R)$-quinacrine; the cytotoxicity of these two stereoisomers was similar. Whether our results with $(S)$-quinacrine offer a meaningful advance in the development of an effective treatment for prion diseases in humans and animals remains to be established.

It is unclear how the stereoselectivity of (S)quinacrine against prions correlates with an earlier observation that only $(R)$-quinacrine was found in urine when a racemic mixture of quinacrine was administered to humans (Hammick and Chambers, 1945). This finding implies that either a receptor binds $(S)$ quinacrine more readily or the pharmacokinetics of the enantiomers differ. In either case, excretion of $(R)$ quinacrine from the body might provide a clue to understanding the difference in antiprion activities of $(S)$ - and $(R)$-quinacrine.

It is reasonable to ask whether $(R)$-quinacrine might be superior to $(S)$-quinacrine in treating prion disease if $(R)$-quinacrine is less toxic than $(S)$ quinacrine in vivo. In this scenario, inhibition of $\mathrm{PrP}^{\mathrm{Sc}}$ formation by $(R)$-quinacrine would occur with fewer complications despite a higher dose of the drug. It will be important to develop an animal model of prion disease to address such questions in vivo. A recent report of experimental CJD in mice failed to show any prolongation of the incubation time following administration of a racemic mixture of quinacrine (Collins et al, 2002).

Studies with racemic quinacrine demonstrated that concentrations in plasma increased rapidly and equilibrated between 30 and $100 \mu \mathrm{g} / \mathrm{L}$, depending on the doses, administration schedules, and species (Armored Medical Research Laboratory and Commission on Tropical Diseases, 1946; Dearborn,

Table 1. Alterations of Tubulin and PrPc Levels in ScN2a Cells Treated with Different Concentrations of Quinacrine

\begin{tabular}{|c|c|c|c|c|c|c|c|c|c|c|}
\hline & \multicolumn{10}{|c|}{ Concentrations of quinacrine $(\mu \mathrm{M})$} \\
\hline & 0 & 0.1 & 0.2 & 0.3 & 0.4 & 0.5 & 0.6 & 0.8 & 1.0 & 1.2 \\
\hline & \multicolumn{10}{|c|}{ Tubulin levels ${ }^{a}$} \\
\hline$(S)$-quinacrine & 1.00 & 0.79 & 0.88 & 0.97 & 0.94 & - & 0.85 & 0.84 & - & 0.78 \\
\hline \multirow[t]{2}{*}{$(R)$-quinacrine } & 1.00 & 1.23 & 1.11 & 0.81 & 1.02 & - & 0.82 & 0.70 & - & 0.85 \\
\hline & \multicolumn{10}{|c|}{ PrP $^{c}$ levels ${ }^{a}$} \\
\hline Generic quinacrine & 1.00 & - & $1.00^{b}$ & - & - & 0.98 & - & - & - & - \\
\hline$(S)$-quinacrine & 1.00 & - & $0.96^{b}$ & - & - & 0.99 & - & - & 1.09 & - \\
\hline$(R)$-quinacrine & 1.00 & - & $1.06^{b}$ & - & - & 0.97 & - & - & 1.17 & - \\
\hline
\end{tabular}

${ }^{a}$ Relative alterations of tubulin and $\mathrm{PrP}^{\mathrm{c}}$ levels were measured by densitometry after Western blot analysis and then normalized by values of untreated $(0 \mu \mathrm{M})$ ScN2a cells.

${ }^{b}$ Measurement at $0.25 \mu \mathrm{M}$. 
1947; Shannon et al, 1944). For eradication of malaria, concentrations of 30 to $50 \mu \mathrm{g} / \mathrm{L}$ in plasma were sufficient, achievable by an oral dose of 100 mg three times daily for several days (Goodman and Gilman, 1955).

Quinacrine is absorbed rapidly, distributed widely, and removed slowly, accumulating in organs and tissues (Armored Medical Research Laboratory and Commission on Tropical Diseases, 1946; Shannon et al, 1944). In vivo, the liver harbors one of the highest concentrations of quinacrine, whereas the brain retains one of the lowest concentrations (Army Malaria Research Unit, 1946; Dearborn et al, 1943). Compared with levels in plasma, quinacrine concentrations can be up to 21,000 times greater in liver (Dearborn, 1947; Goodman and Gilman, 1955; Shannon et al, 1944) and 27.6 times greater in brain (Berliner et al, 1948). From $0.5 \%$ to $3 \%$ of total quinacrine administered to animals was found in the brain (Army Malaria Research Unit, 1946; Blanchard and Schmidt, 1946; Dearborn et al, 1943). Although the brain is one of the areas that exhibit the lowest levels of quinacrine concentration, uptake in brain tissue is significant; the deduced amount available in brain is comparable to the concentrations used to inhibit PrP ${ }^{\mathrm{Sc}}$ propagation completely in ScN2a cells. Quinacrine detected by fluorescence in brain sections of mouse and Guinea pig was distributed specifically in the cerebellum, cortex, hippocampus, and basal ganglia (Crowe and Burnstock, 1984; Howroyd and Gee, 1985).

Both acute and chronic administration of large doses of quinacrine to rats resulted in extensive pathological lesions, including hepatic necrosis, fibrosis, and necrosis of the myocardium and voluntary muscles, and basophilic granules in several tissues (Fitzhugh et al, 1945; Siegel and Mushett, 1944). When therapeutic doses for malaria were administered, with plasma quinacrine levels in excess of $100 \mu \mathrm{g} / \mathrm{L}, 0.2 \%$ to $0.3 \%$ of cases developed toxic psychoses and one individual experienced an epileptiform seizure (Armored Medical Research Laboratory and Commission on Tropical Diseases, 1946; Goodman and Gilman, 1955).

In conclusion, the development of animal models of prion disease in which oral quinacrine treatment can be administered will be important in determining whether or not the potent inhibition of $\mathrm{PrP}^{\mathrm{Sc}}$ demonstrated in ScN2a cultured cells can be extended into animals. Whether one stereoisomer of quinacrine will be found to be more potent in vivo, as is the case in cell culture, is unknown. Additionally, it is unclear whether one enantiomer is preferentially responsible for side effects such a liver toxicity, cardiomyopathy, and toxic psychosis. Although cell cultures provide a reasonable screening system for compounds that inhibit $\mathrm{PrP}^{\mathrm{Sc}}$ formation or enhance its clearance, it is crucial to identify compounds that can be administered long after prion infection has been established and produce a prolongation of the incubation time.

\section{Materials and Methods}

\section{ScN2a Cell Culture and Quinacrine Treatment}

Quinacrine enantiomers were synthesized individually. Synthesis and spectroscopic characterization of the enantiomers are described elsewhere (Craig et al, 1991). Generic quinacrine was obtained from Sigma Chemical and Panorama Compounding. The quinacrine was dissolved in phosphate buffered saline and stored at $4^{\circ} \mathrm{C}$, with light protection during the treatment. A stock solution was prepared freshly every week.

ScN2a cells were cultivated in minimal essential medium with $10 \%$ FCS, 1\% GlutaMax, and $1 \times$ penicillin-streptomycin. For treatments, ScN2a cells were plated to $2 \%$ to $3 \%$ confluency. Various concentrations of generic quinacrine and the enantiomers were added to the cell cultures. Culture media including quinacrine were changed on alternate days during the 6-day treatment. Cells were washed with phosphate buffered saline twice and harvested in an equal volume of lysis buffer (20 mM Tris, $\mathrm{pH} 8.0 ; 150 \mathrm{~mm}$ $\mathrm{NaCl}, 0.5 \% \mathrm{NP} 40,0.5 \%$ deoxycholate, sodium salt). The cell debris was removed by brief centrifugation, and cleared cell lysates were saved at $-20^{\circ} \mathrm{C}$ until used.

\section{PrPsc Detection by Western Blot}

After quantification of the cell lysates, an equal amount of cell lysates was subjected to PK digestion (20 $\mu \mathrm{g} / \mathrm{ml})$ for 1 hour at $37^{\circ} \mathrm{C}$. One hundred $\mu \mathrm{g}$ of PK-digested cell lysates were centrifuged at 16,000 $\times$ $g$ for 1 hour then separated on a $12 \%$ Tris-Glycine SDS-PAGE gel and transferred onto a Protran BA-83 nitrocellulose membrane (Schleicher \& Schuell, Keene, New Hampshire). The membrane was blocked by $5 \%$ nonfat milk. The chimeric human-mouse (HuM) D13 antibody and goat antihuman Fab conjugated to horseradish peroxidase were used as the primary and secondary antibodies, respectively, for the detection of $\mathrm{PrP}^{\mathrm{Sc}}$. An enhanced chemiluminescent (Amersham Pharmacia, Piscataway, New Jersey) substrate for horseradish peroxidase was used for signal development. PrPSc was quantified by densitometry using the Kodak 1D (Kodak, Rochester, New York) application.

\section{PrPsc Detection by ELISA}

PK digestion of cell lysates and centrifugation conditions were the same as described above. The pellet was dissolved and denatured for 1 hour using $6 \mathrm{M}$ guanidine thiocyanate. The denatured lysates were immobilized on the surface of a 96-well Immulon II ELISA plate (Dynex, Chantilly, Virginia) and the rest of the surface in the well was blocked by $3 \%$ BSA. Detection of $\mathrm{PrP}^{\mathrm{Sc}}$ was carried out by a 2-hour incubation of HuM-D18 antibody in $1 \times$ Tris buffered saline followed by 1-hour incubation with goat antihuman Fab conjugated to alkaline phophatase (Pierce, Rockford, Illinois). An additional 1-hour incubation used 1-step pNPP (Pierce), an alkaline phosphatase substrate for the development of a colored product, which 
can be measured at $405 \mathrm{~nm}$. The colorimetric intensity was measured by the microplate reader (Molecular Devices, Sunnyvale, California). A detailed description of this methodology will be described by Peretz et al, manuscript in preparation.

\section{Measurement of Cell Viability}

Cell viability was examined on two different levels: total protein contents and mitochondrial activity. Total protein contents was obtained by multiplication of the volume and the concentration of lysate quantified using a bicinchoninic acid protein quantification kit (Pierce). Mitochondrial activity was measured by the MTT assay. After treatment with quinacrine, the cells were further incubated in fresh media with a final concentration of $0.5 \mathrm{mg} / \mathrm{ml}$ MTT for 3 hours. The insoluble substrates converted by the active mitochondrial dehydrogenases in the surviving cells were released by addition of acidic alcohol $(0.05 \mathrm{~N} \mathrm{HCl}-$ isopropanol) and measured at $570 \mathrm{~nm}$.

\section{Measurement of Tubulin and PrPC Content}

Tubulin and $\operatorname{PrP}^{\mathrm{C}}$ in quinacrine-treated cells were measured by Western blotting followed by densitometry. An equal volume $(20 \mu \mathrm{l})$ of the harvested lysate was separated in an SDS-PAGE gel and transferred to a nitrocellulose membrane. Anti- $\alpha$-tubulin (Zymed, South San Francisco, California) and HuM-D13 antibodies were used for detection for tubulin and $\mathrm{PrP}^{\mathrm{C}}$, respectively. Other conditions and procedures were as described above.

\section{References}

Albert A (1960). Selective toxicity. New York: John Wiley \& Sons.

Armored Medical Research Laboratory and Commission on Tropical Diseases (1946). Plasma quinacrine concentration as a function of dosage and environment. Arch Intern Med 78:64-107.

Army Malaria Research Unit (1946). Mepacrine in animal tissues. Ann Trop Med Parasitol 40:173-180.

Berliner RW, Earle DP, Taggart JV, Zubrod CG, Welch WJ, Conan NJ, Bauman E, Scudder ST, and Shannon JA (1948). Studies on the chemotherapy of the human malarias. J Clin Invest 27:98-107.

Blanchard KC and Schmidt LH (1946). Chemical series of potential interest. In: Wiselogle FY, Wiselogle FY, editors. A survey of antimalarial drugs, 1941-1945. Ann Arbor: J. W. Edwards, 73-175.

Brocks DR and Jamali F (1995). Stereochemical aspects of pharmacotherapy. Pharmacotherapy 15:551-564.

Caspi S, Sasson SB, Taraboulos A, and Gabizon R (1998). The anti-prion activity of Congo red: Putative mechanism. J Biol Chem 273:3484-3489.

Caughey B and Race RE (1992). Potent inhibition of scrapieassociated PrP accumulation by Congo red. J Neurochem 59:768-771.
Collins SJ, Lewis V, Brazier M, Hill AF, Fletcher A, and Masters CL (2002). Quinacrine does not prolong survival in a murine Creutzfeldt-Jakob disease model. Ann Neurol 52: 503-506.

Craig JC, Labelle B, and Ohnsorge U (1991). The absolute configuration of the enantiomers of 6-chloro-9-(4'diethylamino-1'-methylbutyl)-amino-2-methoxyacridine (quinacrine). Chirality 3:436-437.

Crowe R and Burnstock G (1984). Quinacrine-positive neurones in some regions of the guinea-pig brain. Brain Res Bull 12:387-391.

Dearborn EH (1947). The distribution of quinacrine in dogs and in rabbits. J Pharmacol Exp Ther 91:174-177.

Dearborn EH, Kelsey FE, Oldham FK, and Geiling EMK (1943). Studies on antimalarials. J Pharmacol Exp Ther 78:120-126.

Dickinson AG, Fraser H, and Outram GW (1975). Scrapie incubation time can exceed natural lifespan. Nature 256:732733.

Diringer $\mathrm{H}$ and Ehlers $\mathrm{B}$ (1991). Chemoprophylaxis of scrapie in mice. J Gen Virol 72:457-460.

Doh-ura K, Iwaki T, and Caughey B (2000). Lysosomotropic agents and cysteine protease inhibitors inhibit scrapieassociated prion protein accumulation. J Virol 74:48944897.

Ehlers B and Diringer H (1984). Dextran sulphate 500 delays and prevents mouse scrapie by impairment of agent replication in spleen. J Gen Virol 65:1325-1330.

Findlay GM (1951). Recent advances in chemotherapy. Philadelphia: The Blakiston Company.

Fitzhugh OG, Nelson AA, and Calvery HO (1945). The chronic toxicity of quinacrine (atabrine). J Pharmacol Exp Ther 85: 207-221.

Goodman LS and Gilman A (1955). The pharmacological basis of therapeutics. New York: Macmillan.

Hammick DL and Chambers WE (1945). Optical activity of excreted mepacrine. Nature 155:141.

Howroyd PC and Gee DJ (1985). The use of fluorescence microscopy and microfluorimetry to study the distribution of quinacrine in mice. Histochem J 17:13-21.

Hutt AJ and Tan SC (1996). Drug chirality and its clinical significance. Drugs 52:1-12.

Kimberlin RH and Walker CA (1983). The antiviral compound HPA-23 can prevent scrapie when administered at the time of infection. Arch Virol 78:9-18.

Korth C, May BCH, Cohen FE, and Prusiner SB (2001). Acridine and phenothiazine derivatives as pharmacotherapeutics for prion disease. Proc Natl Acad Sci USA 98:9836-9841.

Peretz D, Williamson RA, Kaneko K, Vergara J, Leclerc E, Schmitt-Ulms G, Mehlhorn IR, Legname G, Wormald MR, Rudd PM, Dwek RA, Burton DR, and Prusiner SB (2001). Antibodies inhibit prion propagation and clear cell cultures of prion infectivity. Nature 412:739-743.

Priola SA, Raines A, and Caughey WS (2000). Porphyrin and phthalocyanine antiscrapie compounds. Science 287:15031506.

Shannon JA, Earle DP, Brodie BB, Taggart JV, and Berliner RW (1944). The pharmacological basis for the rational use of 
Atabrine in the treatment of malaria. J Pharmacol Exp Ther 81:307-330.

Siegel H and Mushett CW (1944). Structural changes following administration of quinacrine hydrochloride. Arch Pathol 38:63-70.

Supattapone S, Nguyen H-OB, Cohen FE, Prusiner SB, and Scott MR (1999). Elimination of prions by branched polyamines and implications for therapeutics. Proc Natl Acad Sci USA 96:14529-14534.

Supattapone S, Wille H, Uyechi L, Safar J, Tremblay P, Szoka FC, Cohen FE, Prusiner SB, and Scott MR (2001). Branched polyamines cure prion-infected neuroblastoma cells. J Virol 75:3453-3461.
Tucker GT (2000). Chiral switches. Lancet 355:1085-1087.

Webster RV, Craig JC, Shyamala V, Kirby GC, and Warhurst DC (1991). Antimalarial activity of optical isomers of quinacrine dihydrochloride against chloroquine-sensitive and -resistant Plasmodium falciparum in vitro. Biochem Pharmacol 42(Suppl):225-227. 